\title{
Reliability of Echocardiography Measurement of Patent Ductus Arteriosus Minimum Diameter: A Meta-analysis
}

\author{
Yong-quan Huang ${ }^{1}$, Yin Huang ${ }^{1}$, Dan Huang ${ }^{2}$, Mao Liu $^{2}$, Li-yun Luo ${ }^{1}$, Jian Chen ${ }^{1, *}$ \\ ${ }^{1}$ Department of Ultrasound, The Fifth Affiliated Hospital of Sun Yat-sen University, China \\ ${ }^{2}$ Department of Cardiovascular, The Fifth Affiliated Hospital of Sun Yat-sen University, China
}

Copyright $\bigcirc 2016$ by authors, all rights reserved. Authors agree that this article remains permanently open access under the terms of the Creative Commons Attribution License 4.0 International License

\begin{abstract}
Objective: To systematically evaluate the reliability of transthoracic echocardiographic (TTE) measurements of the narrowest diameter in patients with patent ductus arteriosus (PDA) in comparison to angiographic measurements. Methods: We searched articles published between January 1990 to August 2015 that compared diameters of PDA both measured by TTE and angiography in PubMed, Cochrane Library, Wanfang Data database, and CNKI. Two researchers screened the literature, extracted data and assess the quality independently. Weighted mean difference (WMD) and 95\% confidence intervals $(95 \% \mathrm{CI})$ were calculated, heterogeneity was evaluated, and publication bias was estimated. The correlation coefficients of the narrowest PDA diameter measured by echocardiography and angiography were merged by RevMan 5.2 and Stata 12.0 software. Results: 11 articles involving 870 patients are included. Meta-analysis demonstrated that measurement values of the minimum inner diameter by TTE were greater compared to angiography measurements (WMD $=0.86,95 \% \mathrm{CI}: 0.71 \sim 1.02, \mathrm{P}<0.001$ ). Echocardiographic and angiographic measurements correlated closely with each other $(\mathrm{R}=0.75,95 \% \mathrm{CI}$ : $0.52 \sim$ 0.97). There was no evidence of publication bias. Conclusions Measurement of the minimum inner diameter of PDA by TTE has value for both the selection of therapeutic strategy and the PDA occluder model. Nevertheless, echocardiography overestimates minimum diameter in comparison to angiography.
\end{abstract}

Keywords Patent Ductus Arteriosus, Echocardiography, Occlusion, Cardioangiography, Meta-analysis

\section{Introduction}

Patent ductus arteriosus (PDA) is the second most common form of congenital heart disease [1]. It is found in $15 \%-21 \%$ of patients with congenital heart disease, with females affected twice as common as men. At present transcatheter occlusion is an established, minimally invasive treatment options for small and moderate size PDAs, particularly if the minimum ductal luminal diameter is smaller than $4 \mathrm{~mm}$ [2]. For planning of transcatheter PDA closure including selection of appropriate device, accurate measurements of the minimal luminal diameter are critical. Invasive angiography is considered the reference standard for determination of ductal size and morphology. But it is invasive, expensive, and associated with a small risk of complications. In contrast, transthoracic echocardiography (TTE) is a non-invasive, less expansive, and widely available alternative for ductal measurement. Nevertheless, studies comparing these two methods have reported discordant results [3-5]. Therefore, we conducted a meta-analysis to compare the minimum diameter of PDA measured both by TTE and angiography and evaluate the clinical significance of TTE measurements for choice of treatment and PDA occluder model.

\section{Materials and Methods}

\subsection{Literature Search Strategy}

We searched PubMed, Cochrane Library, Wanfang data, and China National Knowledge Internet (CNKI) for published data comparing TTE and angiography two measurement of the minimum diameter of PDA. Key words used in the literature search were 'patent ductus arteriosus', 'transcatheter closure', 'transcatheter occlusion', 'angiocardiography', 'ultrasonography', and 'echocardiography'. The search was limited to studies in English and Chinese language from January 1990 to June 2015. No other restrictions were applied. We also manually searched the reference lists of included articles for original 
reviews, and searched those for additional relevant studies.

\subsection{Study Inclusion and Exclusion Criteria}

Studies were included for statistical analysis if they fulfilled the following criteria: human subjects with at least 20 subjects included, perspective or retrospective design, reported measurement value of both mean and standard deviation of the minimum inner diameter of the PDA measured by TTE and angiography. We excluded articles which were reviews, abstracts, letters to editor, editorial reports, duplicate publications, and articles of which the full text was not available. When there were several articles about the same cohort, the article reporting the largest number of cases was selected.

\subsection{Quality Assessment and Data Extraction}

Two authors (Huang YQ and Huang Y) participated in the assessment of the quality of cohort studies included in this meta-analysis by using a modified Newcastle Ottawa Scale (NOS), which graded the quality of a study on a scale from 0 to 9 , depending on patient selection, comparability of TTE and angiographic measurements, and exposure assessment. Articles exceeding a score of 8 were considered as high quality and those with a score of 5-7 were considered as moderate quality. Any disagreement was resolved by consensus or assistance from the third author (Liu M).

\subsection{Statistical Analysis}

We used the software RevMan 5.2 and Stata 12.0 to perform all the statistical analysis. Results of combination were expressed as weight mean difference (WMD). Afterwards $95 \%$ confidence intervals $(95 \% \mathrm{CI})$ were also calculated to indicate the precise of above effect measures and forest plot was drawn. Heterogeneity was assessed with the Q-test and the $\mathrm{I}^{2}$ statistic. Low level of heterogeneity was defined as $\mathrm{I}^{2}$ value $\leqq 50 \%$. Pooled estimates of WMD were calculated using the fixed-effects model if no substantial heterogeneity existed; otherwise, the random-effects model was used. Publication bias was evaluated using a funnel plot.

\section{Results}

\subsection{Identification of Eligible Studies}

The search strategy identified 2283 non-duplicated related studies (PubMed 697, Cochrane library 14, CNKI 791,
Wanfang data 781). After exclusion of 2247 studies, 36 studies were examined at length. Finally, 11 case control studies were eligible for inclusion criteria, 10 of which were written in Chinese and 1 was written in English (Figure 1).

\subsection{Essential Characteristics of Eligible Studies and Quality Assessment}

The baseline characteristics of the 11 eligible studies included in our analysis are demonstrated in Table 1. Of these included 11 studies [3, 4, 6-14], 10 were performed in China and 1 was conducted in India. All studies had a retrospective design. Together they included 870 PDA patients with an age range from1 to 67 . Results of quality assessment of these 11 eligible studies showed that these studies had high quality according to the NOS criteria (Table 1).

\subsection{Comparison of the Minimum Inner Diameter Value Measured by TTE and Cardiaoangiography}

All the 11 studies reported the minimum inner diameter value measured by both TTE and angiography. Heterogeneity test demonstrated that there was no obvious heterogeneity $\left(\mathrm{Q}=11.07, \mathrm{P}<0.001, \mathrm{I}^{2}=0.00 \%\right)$. Pooled estimates which were calculated with the fixed-effects model demonstrated that the minimum inner diameter of PDA measured by TTE was larger than that measured by angiography. (WMD $=0.86,95 \% \mathrm{CI}$ : 0.71-1.20, $\mathrm{P}<0.001$ ). (Figure2)

\subsection{Correlation between the Minimum Inner Diameters Measured by TTE and by Angiography}

Four studies reported the correlation between the minimum inner diameter measured by TTE and angiography. The coefficients were: $0.44,0.86,0.82,0.87$. Heterogeneity test demonstrated that there was obvious heterogeneity $\left(\mathrm{I}^{2}=97.2 \%, \mathrm{P}<0.001\right)$. Pooled estimates using Random-effect model calculated that the correlation coefficient was 0.75 , 95\%CI: 0.52 0.97(Figure 3)

\subsection{Publication Bias}

Begg's rectify rank correlation test and Egger's linear regression did not find publication bias among the studies (Begg's $\mathrm{P}=0.876$, Egger's $\mathrm{P}=0.520$ ) .We can also directly draw the same conclusion through the funnel plot (Figure 4). 


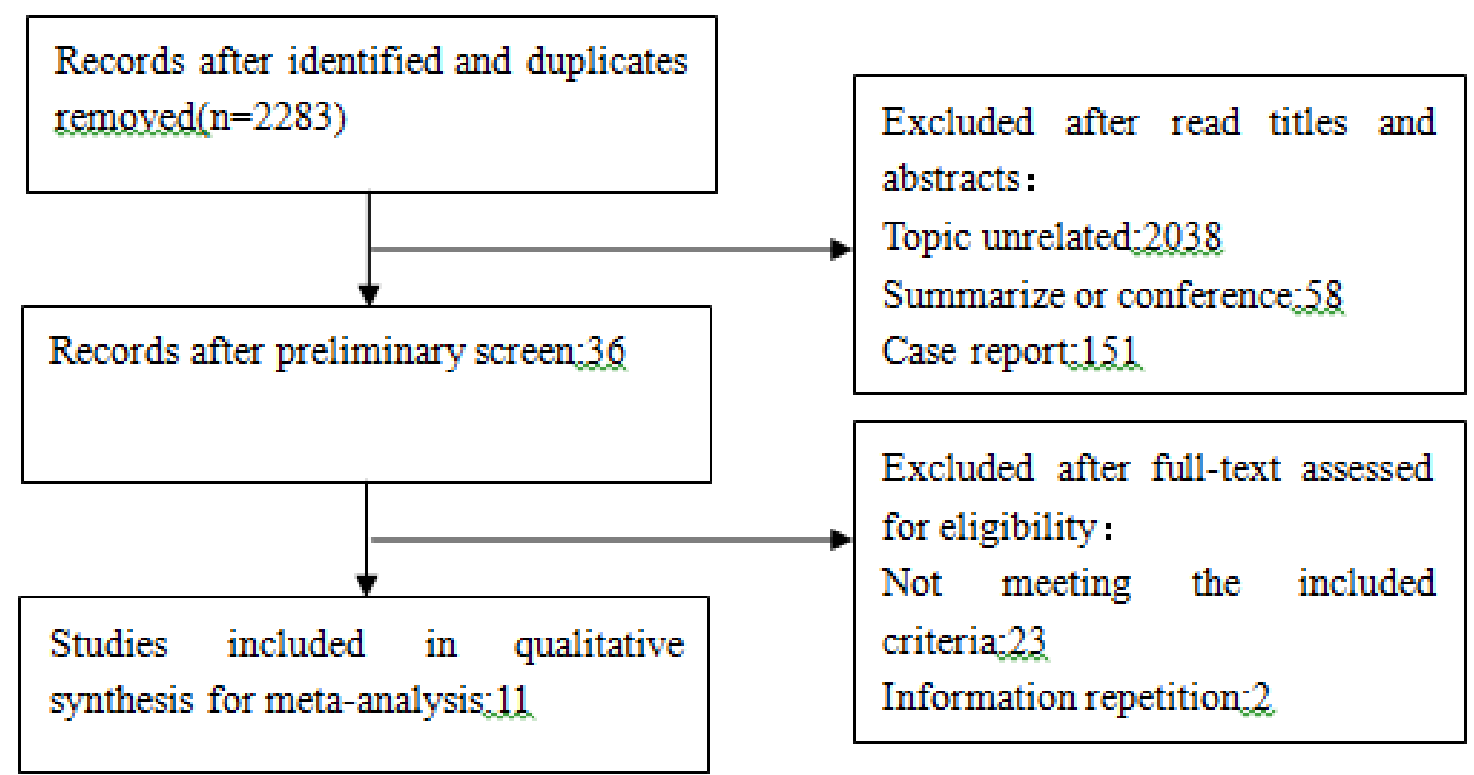

Figure 1. Flow chart of study selection

Table 1. Data extracted from included studies and quality assessment of included studies

\begin{tabular}{|c|c|c|c|c|c|}
\hline Studies & Country & Study sample & Males, $\%$ & Mean Age, year & NOS \\
\hline Wong, J.A. 1998 & India & 27 & - & - & 7 \\
\hline Zhang, Q Q 2001 & China & 262 & 14 & $14.6 \pm 13.6$ & 8 \\
\hline Wang, Sh X 2002 & China & 30 & 13 & $13.2 \pm 12.3$ & 8 \\
\hline Peng, Y F 2003 & China & 45 & 26 & $14.0 \pm 14.7$ & 8 \\
\hline Wang, T J 2006 & China & 41 & 21.95 & $6.15 \pm 4.0$ & 7 \\
\hline Fan, W F 2007 & China & 132 & 54.55 & - & 8 \\
\hline Liu, T Ch 2008 & China & 61 & 21 & - & 8 \\
\hline Bian, H L 2008 & China & 52 & 22 & $4.86 \pm 4.43$ & 7 \\
\hline Zhang, X Jun 2010 & China & 96 & - & 3.7 & 7 \\
\hline He, H 2012 & China & 74 & - & - & 7 \\
\hline Lin, F H 2012 & China & 50 & - & & 7 \\
\hline
\end{tabular}

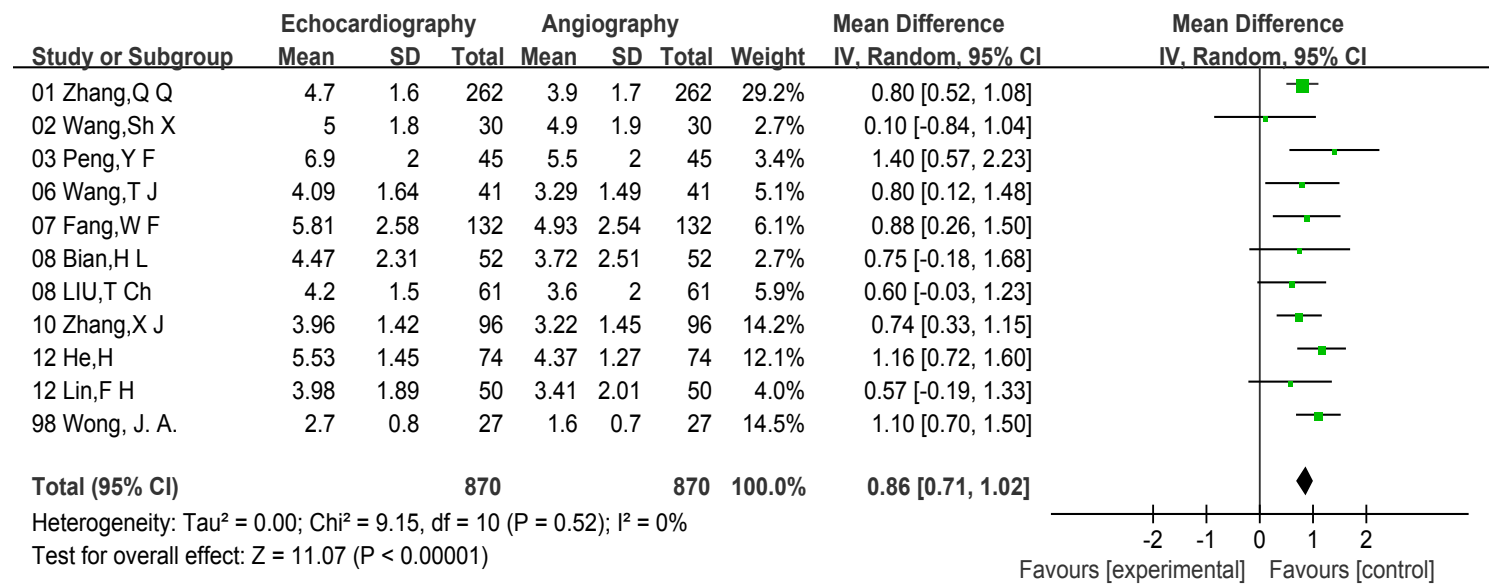

Figure 2. Forest map of the comparison of the minimum inner diameter value measured by TTE and angiography (units for Mean and SD: mm) 


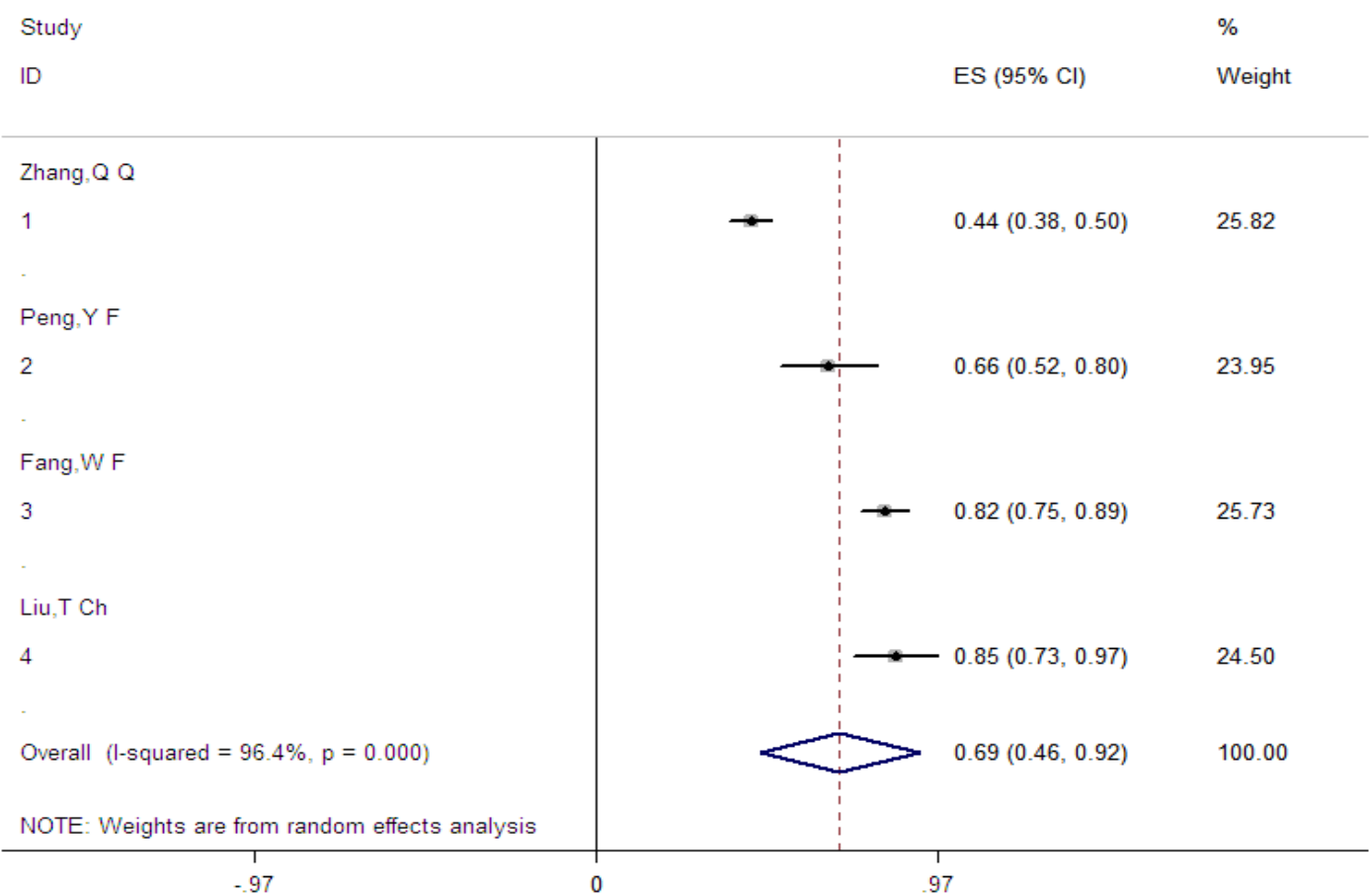

Figure 3. Pooled estimate the correlationship between the minimum inner diameter measured by TTE and angiography

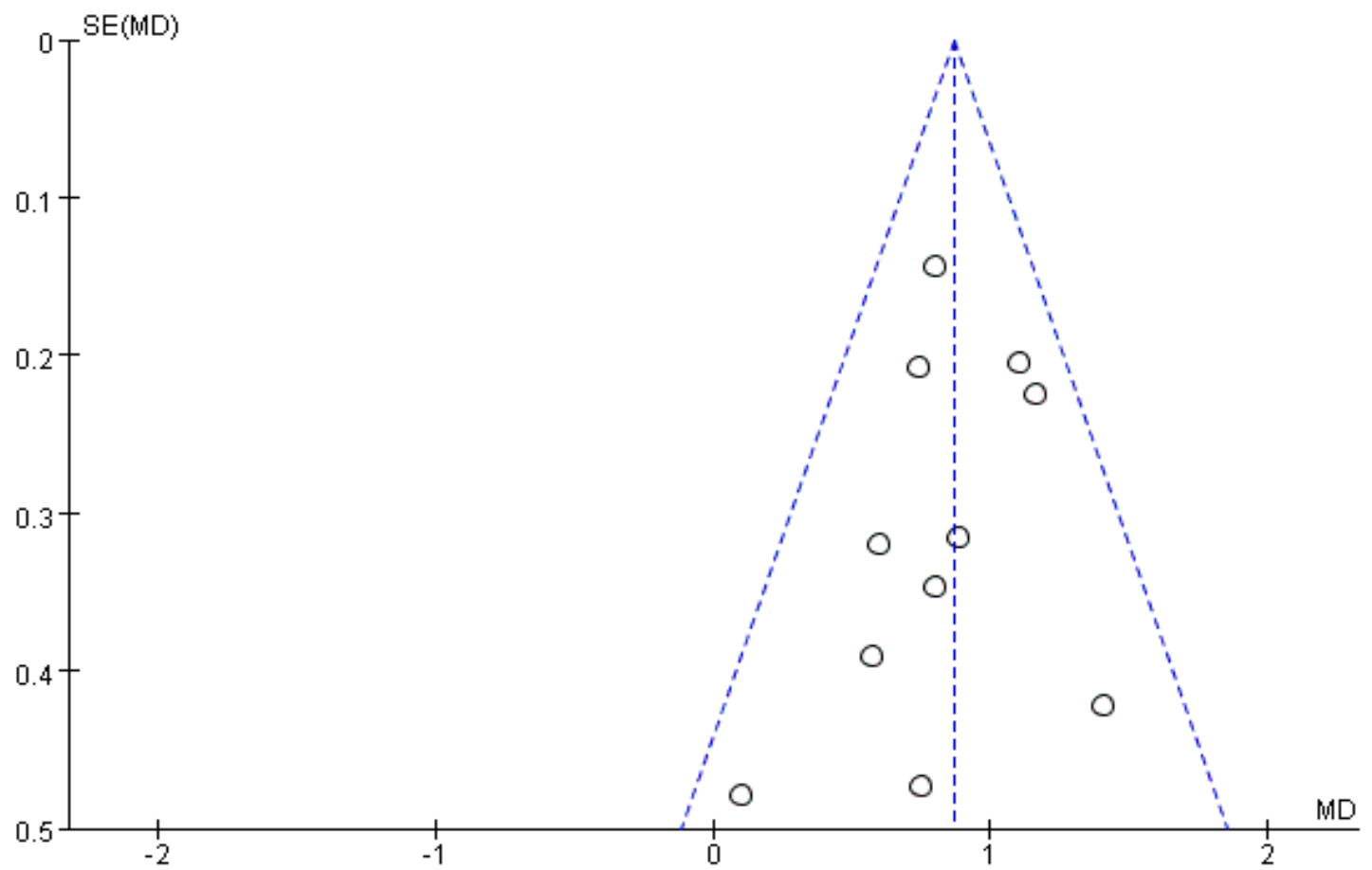

Figure 4. Funnel map to assess the publication bias of the included articles

\section{Discussion}

PDA is one of the most common forms of congenital heart disease, and should be treated once a definitive diagnosis is established. Transcatheter PDA occlusion is characterized by high success rate, efficacy and minimally invasiveness. It should be the first choice of treatment of PDA [15]. TTE which is noninvasive, easily performed, inexpensive, and well tolerated by the patient, is widely used as the primary method of screening and follow-up examinations before and after PDA closure. It provides precise measurement and anatomy morphology of the PDA. Angiography, is 
considered the reference standard, but is more expensive, invasive and associated with a small risk of complications.

According to the results of our meta-analysis, measurements of the minimum inner PDA diameter by TTE were larger than that measured by angiography. This may be due to the number of factors: 1) Available views with two dimensional echocardiographic views are limited by bony structures of the chest wall and lung tissue. It is therefore challenging to define the shape of the PDA, which is highly variable between patients. Assessment can be further limited by lateral wall echo drop-out. Together these two factors both can result in obscured display of the inner wall of the PDA and lead to the higher measurement values. Color Doppler Flow Imagine (CDFI) can also cause higher measurement values if the gain parameter is improperly set. On the other hand, angiography also has well defined limitations, which could lead to underestimation of luminal measurements. Prior reports describe spasm of the ductus arteriosus during angiography $[10,16,17]$.We conclude that measurement of the minimum inner diameter of PDA by TTE can provide guidance for choosing therapeutic approach and device type if transcatheter closure is selected.

There are several potential limitations of this meta-analysis. First, ultrasound machines used and skill of operators were different among the included studies, which could lead to measurement bias. Second, the majority of patients included were from China and India, which could lead to selective reporting bias. Third, besides English and Chinese, papers in other languages such as German, French, Spanish and Italian was omitted, which could lead to a language bias. Therefore further prospective trials will be necessary.

\section{Conclusions}

Measurement of the minimum inner diameter of PDA by TTE has value for both the selection of therapeutic strategy and the PDA occluder model. Nevertheless, echocardiography overestimates minimum diameter in comparison to angiography, which surgeons should take in mind when decide choice of suitable treatment strategy.

\section{REFERENCES}

[1] Hammerman, C. and M. Kaplan, Oxygen saturation during and after feeding in healthy term infants. Biol Neonate, 1995. 67(2): p. 94-9.

[2] Glaus, T. M., et al., Catheter closure of patent ductus arteriosus in dogs: variation in ductal size requires different techniques. J Vet Cardiol, 2003. 5(1): p. 7-12.
[3] Zhang, Q Q., et al., Comparative study of minimum diameter and morphology of patent ductus arteriosus between echocardiography and angiography. Chinese Journal of Medical Imaging Technology, 2001. 17(10): p. 975-977.

[4] Wang, Sh X., et al., Method of Amplatzer of patent ductus arteriosus occlusion- the feasibility of measurement value of echocardiography. Chinese Journal of Critcal Care Medicine, 2002(05): p. 42-43.

[5] Shi, H., et al., Study of adult patent ductus arteriosus with transthoracic and transesophageal multiplanar echocardiography and balloon-catheter angiography. Chinese Journal of Medical Imaging, 2006. 14(6): p. 419-421.

[6] Wong, J.A., et al., Validation of color Doppler measurements of minimum patent ductus arteriosus diameters: significance for coil embolization. Am Heart J, 1998. 136(4 Pt 1): p. 714-7.

[7] Peng,Y F., et al., Catheter Closure of Patent Ductus Arteriosus Using Amplatzer Duct Occluder: Clinical Results. Journal of Fujian Medical University, 2003. 37(4): p. 397-400.

[8] Wang,T J., Clinical study of interventional treatment for common congenital disease in children. 2006, Zhengzhou University.

[9] Fan,W F., et al., The study of the selected value of Ultrasound examination and angiography in the patent ductus arteriosus. Clinical Focus, 2007. 22(13): p. 951-952.

[10] Liu, T Ch., et al., Comparison between transthoracic echocardiography and cardiac catheterization in measuring diameter of patent ductus arteriosus and accessing pulmonary artery pressure. South China Journal of Cardiovascular Diseases, 2008. 14(1): p. 23-25.

[11] Bian, H L., Interventional therapy of congenital heart disease clinical research. 2008, Suzhou University.

[12] Zhang, X J., et al., The combined use of cardioangiography and ultrasonography in interventional therapy for congenital heart disease in children. Journal of Interventional Radiology, 2010. 19(3): p. 177-180.

[13] He, H., et al., The combined application value of ultrasonography and cardioangiography in interventional treatment for patients with congenital heart disease. Chinese Journal of Clinical Healthcare, 2012. 15(1): p. 24-26.

[14] Lin, F H. and Zhou, F J., Significance of cardioangiography in interventional treatment of congenital heart disease. China Health Care Nutrition, 2012. 22(12): p. 5461-5462.

[15] Gao, M. and Hou, Y L., Interventional treatment of patent ductus arteriosus. Shandong Medical journal, 2009. 49(9): p. 106.

[16] Jiang, Sh L., Dai, R P. and Zhao, Sh H., Application of Amplatzer occluder in treatment of patent ductus arteriosus, Editors. 1999. p. 745.

[17] Bian, H L. and Yang, X J., Comparative Study on Different Methods for Measurement of Diameter of Patent Ductus Arteriosus in Transcatheter Interventions. Journal of Applied Clinical Pediatrics, 2008. 23(1): p. 44-45. 